\title{
Empleo y sismo: la situación ocupacional de la población damnificada*
}

\author{
José Benigno Morelos* *
}

En el trabajo se describe la situación ocupacional de la población damnificada, haciendo referencia a la escolaridad, nivel de ingreso, edad y rama de actividad de los ocupados y estimando los niveles de desempleo como consecuencia del sismo. Como contexto de los niveles de empleo se repasan aspectos del desarrollo urbano desde la perspectiva demográfica, para lo cual se analizan las tasas de crecimiento poblacional de las distintas unidades urbanas, se establece la clasificación de las delegaciones según categoría migratoria y se mencionan algunos rasgos del envejecimiento de la población en la ciudad central. Finalmente, se concluye con algunas consideraciones sobre el empleo urbano.

\section{Introducción}

Entre las múltiples actividades que realizaron las instituciones de educación superior a raíz del sismo del 19 de septiembre de 1985, destacan las investigaciones de corte socioeconómico. Entre éstas, se encuentra el estudio que efectuó el Instituto de Investigaciones Sociales de la Universidad Nacional Autónoma de México con el apoyo del Departamento del Distrito Federal; su objetivo consistió en recolectar información sobre las principales características demográficas, económicas y sociales de los damnificados para establecer, en función de las variables seleccionadas, el perfil de los mismos.

Para tal efecto se diseñó y levantó una encuesta durante el periodo que fue del 28 de septiembre al 7 de octubre del mismo año. Como marco muestral se utilizó el listado de albergues y campamentos del Distrito Federal proporcionado por la Secretaría General de Desarrollo Social. De acuerdo con el padrón oficial, el número de personas que se encontraba en esos lugares durante el periodo de referencia era de aproximadamente 30 mil, $73 \%$ de los cuales se ubicaba en albergues y el restante $27 \%$ en campamentos.

De este numeroso grupo se entrevistó a 16630 personas, cifra que

* Una versión preliminar de este trabajo se presentó en el Coloquio: “El sismo y sus efectos", organizado por el-Instituto de Investigaciones Sociales y la Coordinación de Humanidades de la Universidad Nacional Autónoma de México, y la Universidad de Berkeley, el 17 y 18 de septiembre en la Ciudad Universitaria, México D.F.

* * Profesor investigador del Centro de Estudios Demográficos y de Desarrollo Urbano (CEDDU) de El Colegio de México y asesor del C. jefe del Departamento del Distrito Federal. Las opiniones vertidas en este trabajo son responsabilidad exclusiva del autor. 
representa $55 \%$ del total de damnificados (Rabell y Mier y Terán, 1986). Por el tamaño de la muestra, se considera que los datos obtenidos son representativos de la población bajo estudio, por lo que se pueden formular generalizaciones para el total de la población que temporalmente se encontraba instalada en esos sitios debido a que su vivienda había resultado destruida, dañada o bien se encontraba en zona de peligro; en cierta medida, también pueden hacerse generalizaciones sobre aquellos habitantes de la ciudad de México (más precisamente, con los de las delegaciones Cuauhtémoc y Venustiano Carranza), que poseen un perfil similar al de los afectados por el terremoto. En las dos circunscripciones mencionadas residía 91\% de los entrevistados y cerca de 8 mil personas declararon tener su vivienda en las colonias Morelos y Centro que se localizan en el perímetro de la Cuauhtémoc.

Si se considera el tipo de vivienda, tenencia, número de cuartos, hacinamiento y las características sociales de los damnificados (Rabell y Mier y Terán, 1986), se está frente a un estrato de la sociedad capitalina que podría situarse en los niveles intermedios y bajos de la escala social.

La inserción de los damnificados en dichos estratos se explica en buena medida por las modalidades del desarrollo capitalista del país, uno de cuyos rasgos distintivos es la heterogeneidad estructural y ocupacional de la mano de obra, que determina la presencia de mercados formales e informales de fuerza de trabajo.

En esta perspectiva, y dada la naturaleza de la información recabada, en el presente trabajo se describirá la situación ocupacional de los damnificados durante la primera quincena de septiembre y el impacto que tuvo la actividad sísmica en el empleo.

Tres apartados integran el estudio. En el primero se repasan los rasgos principales del desarrollo metropolitano, básicamente desde la óptica demográfica; en el segundo se alude al análisis de los niveles de empleo y desempleo, y en el tercero se hacen algunas consideraciones sobre la problemática del empleo urbano.

1

Durante las últimas décadas, el desarrollo económico de la ciudad de México ha sido la resultante del dinamismo industrial apoyado en la estrategia de sustitución de importaciones, que ha alentado y condicionado la evolución urbana del país. Al fenómeno de la centralización se añade en México el de la metropolización, que da paso a la suburbanización en sus dos modalidades: desbordamiento de la población hacia las delegaciones periféricas a la ciudad central y hacia los municipios del Estado de México. ${ }^{1}$

${ }^{1}$ En sentido estricto, el concepto de metropolización se refiere a la ubicación 
De acuerdo con el ciclo vital de las aglomeraciones urbanas, se distinguen cuatro fases de desarrollo: urbanización, suburbanización, desurbanización y reurbanización (Korcelli, 1984). En la etapa de urbanización el crecimiento de la ciudad central predomina sobre la periferia, pero en la de suburbanización el crecimiento de la periferia es el más dinámico. En el periodo 1950-1960, las tasas de crecimiento de la ciudad central, Distrito Federal y área metropolitana de la ciudad de México (AMCM), eran de 2.3, 4.6 y 5.5 por ciento respectivamente. En los años setenta los valores correspondientes fueron de $-1.1,2.2$ y 5.5 por ciento.

De manera parecida, se han dado cambios en la importancia relativa de las poblaciones de las unidades urbanas respecto a la población del país. Hasta 1950, la proporción de la población de la ciudad central en relación a la población del país observó aumentos sucesivos. De ese año en adelante descendió; en 1980 el valor fue de $4.0 \%$. Por su parte, el Distrito Federal muestra un cambio en la tendencia de este indicador a partir de 1970, mientras que el AMCM ve incrementada su participación durante todo el periodo (véase el cuadro 1).

\section{CUADRO 1}

Población total según distintas unidades territoriales (cifras en miles)

\begin{tabular}{|c|c|c|c|c|c|c|}
\hline & 1930 & 1940 & 1950 & 1960 & 1970 & 1980 \\
\hline 1. País & 16552 & 19653 & 25791 & 34923 & 48225 & 66846 \\
\hline 2. Ciudad central & 1029 & 1148 & 2249 & 2829 & 3002 & 268 \\
\hline 3. Distrito Federal & 1229 & 1757 & 3050 & 4870 & 6874 & 88 \\
\hline $\begin{array}{l}\text { 4. Área metropolitana de la ciu- } \\
\text { dad de México }\end{array}$ & 1050 & 1560 & 2872 & 4910 & 8355 & 1427 \\
\hline (2) / (1) x 100(\%) & 6.2 & 7.4 & 8.7 & 8.1 & 6.2 & 4. \\
\hline 6. (3) $/(1) \times 100(\%)$ & 7.4 & 8.9 & 12.6 & 14.8 & 15.2 & 13.7 \\
\hline 7. (4) /(1) $\times 100(\%)$ & 6.3 & 7.9 & 11.1 & 15.1 & 17.3 & 31. \\
\hline
\end{tabular}

Fuente: Censos Generales de Población DGE (SIC-SPP) y Maria Eugenia Negrete y Héctor Salazar, “Dinámica de crecimiento de la población de la ciudad de México: 1900-1980" (por aparecer en Atlos de lo ciudad de México, El Colegio de México/DDF).

de la población en un área político-administrativa distinta a la ciudad central; en sentido más laxo, alude al movimiento de la población hacia zonas periféricas sean o no distintas desde el punto de vista político-administrativo. En este artículo se hace referencia a ambos aspectos, aunque las fechas en que se presenta la metropolización varía según el criterio que se utilice. En el primer caso, la metropolización se inicia en los años cincuenta y, de manera intensa, a partir de 1957 con la fundación de Ciudad Satélite (Tarres, 1986). En la segunda acepción, el comienzo se fija en los años cuarenta, cuando se empiezan a poblar las delegaciones al sur de la ciudad (Negrete y Salazar, 1986). El lugar central hace referencia a la ciudad de México constituida actualmente por el área y la población de las delegaciones Cuauhtémoc, Benito Juárez, Miguel Hidalgo y Venustiano Carranza. 
Tanto el comportamiento de las tasas de crecimiento como el de las proporciones de población de las unidades urbanas aquí considerados, tipifican la gradual desconcentración de la población del área central. A nivel nacional se han verificado transformaciones en los niveles de crecimiento de las áreas metropolitanas y de las ciudades medianas y pequeñas (Graizbord, 1986), lo cual concuerda con lo señalado por Korcelli. En un estudio reciente (Negrete y Salazar, 1986), se documentan los cambios en la estructura urbana del país desde la óptica del desarrollo metropolitano y se señala que "los municipios centrales de la zona metropolitana han ido concentrando proporciones menores de población frente a una importancia creciente de sus periferias correspondientes".

En el interior del Distrito Federal se han producido alteraciones en los patrones de asentamiento poblacional, debido a los cambios de residencia. Aunque la información es indirecta, la clasificación de las delegaciones según su calidad migratoria a partir de 1950, pone de manifiesto lo anterior. En los años setenta las circunscripciones que conforman la ciudad central se encuentran en el grupo de fuerte rechazo - todas con tasas negativas de crecimiento (véase cuadro 2). Por el contrario sólo Coyoacán e Iztapalapa se han mantenido en el grupo de fuerte atracción. En general el paso de una categoría a otra se presenta en forma gradual, ya sea que el movimiento sea de equilibrio a débil rechazo o de equilibrio a débil atracción. Como excepciones estan Xochimilco, que de débil rechazo pasó a débil atracción, e Iztacalco, que de fuerte atracción se trasladó al grupo de débil rechazo (véase cuadro 2).

En lo que se refiere al Distrito Federal en su conjunto, de entidad de fuerte atracción en los años cincuenta se convierte en área de equilibrio en los setenta y con una tendencia clara a transformarse en área de rechazo; tales categorías coinciden con la fase de centralización y suburbanización.

Por otra parte, la dinámica de crecimiento diferencial intraurbano, pro-' ducto de la desconcentración de la población de la ciudad central hacia la periferia, es indicativa de los cambios que están ocurriendo en los patrones migratorios: ciudad-ciudad y ciudad-campo, ${ }^{2}$ lo cual se considera como una de las nuevas manifestaciones del desarrollo urbano del país (Graizbord, 1986). En paralelo se ha evidenciado la pérdida de población o despoblamiento de la ciudad central, que entre 1970 y 1980 registró una disminución de 314 mil habitantes. ${ }^{3}$ Razones de tipo económico y social

${ }^{2}$ Hasta ahora se sabe poco de los movimientos o la movilidad espacial de la población al interior del Distrito Federal y Амсм. Es posible que la población de las delegaciones centrales se haya reubicado en las delegaciones del sur y poniente o en los municipios metropolitanos - Naucalpan, Huixquilucan, Tlalnepantla-, con lo cual se estaría verificando la migración, ciudad central-periferia, algo que puede considerarse como migración del tipo ciudad-ciudad.

${ }^{3}$ De las cuatro delegaciones centrales, la Cuauhtémoc viene perdiendo población desde 1960. Entre 1970 y 1980, la reducción fue de 120 mil habitantes, cifra 
EMPLEO Y SISMO

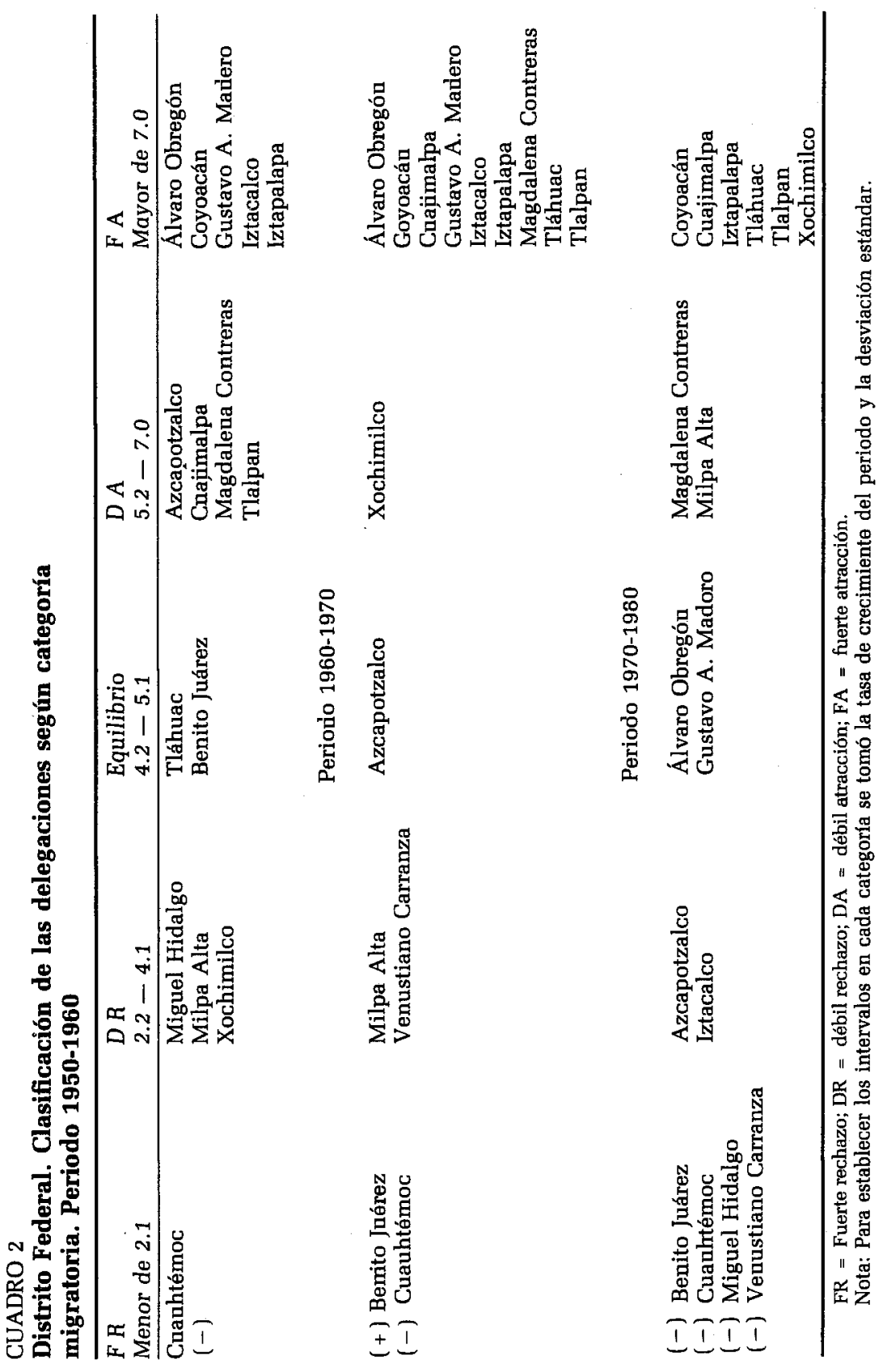


como los cambios en los usos del suelo urbano, la especulación inmobiliaria, los subsidios al transporte urbano de la ciudad y la movilidad social que ha propiciado la constitución y el fortalecimiento de la clase media capitalina, explican la segregación espacial y social en el AMCM. Autores como Garza y Schteingart (1984) hacen énfasis además de en la movilidad social, en el gasto público, los programas de vivienda por parte del Estado y la invasión-regularización como determinantes de ambos tipos de segregaciones.

Una característica adicional de la población de la ciudad central es su gradual envejecimiento y el predominio relativo de la población femenina. En 1950, la proporción de población mayor de 65 años era de $3.5 \%$ en la ciudad central. ${ }^{4}$ En 1980 se elevó a 5.8\%. En este año los valores de dicho indicador para el Distrito Federal y el AMCM fueron de 3.3 y 3.4 por ciento respectivamente. En la ciudad central, el porcentaje de mujeres ha sido en los últimos 30 años del orden de $54 \%$, dos y tres puntos porcentuales mayor que los correspondientes al Distrito Federal y al AMCM.

Tanto el envejecimiento como la relativa sobrepoblación femenina son resultado de la migración, la mortalidad diferencial - mayor la de los hombres que la de las mujeres- y la disminución de la fecundidad. Hasta 1960, la ciudad central fue un área de inmigración, pero después de ese año se ha convertido en una de expulsión. Aquí se combinan dos tipos de migración neta, que probablemente influye aunque de manera aun no determinada en el crecimiento de la población senecta. Igualmente la selectividad de migrantes favorece el predominio relativo de mujeres; al combinarse con la mortalidad, este factor explica la mayor proporción de mujeres en la ciudad central que en el resto del AMCM. ${ }^{5}$

Desde la perspectiva económica, los cambios más importantes son los relativos a la concentración industrial. Hasta 1970 su ritmo fue creciente,

que representa $38 \%$ del total. Las tres delegaciones restantes muestran disminuciones en sus volúmenes a partir de 1970 .

${ }^{4}$ Las delegaciones Cuauhtémoc y Benito Juárez son las que muestran los mayores porcentajes de población mayor de 65 años y, correlativamente, las menores proporciones de población de 15 años y menos. En 1980, el primer indicador para esas delegaciones registró los valores de 6.7 y 6.6 por ciento, respectivamente, y para el segundo de 27.1 y 29.6 por ciento. La proporción de mujeres en la Cuauhtémoc y Benito Juárez durante los últimos 40 años ha fluctuado entre 53.7 y $\mathbf{5 5 . 7}$ por ciento.

5 Stern y Corona (1985), en un estudio comparativo sobre las poblaciones de Hidalgo y el Distrito Federal, proporcionan evidencia del efecto que tiene la migración en la conformación de la estructura por edad. Igualmente atribuyen a la selectividad (mujeres) de la migración, la mayor presencia de las mismas en los grupos de edad donde la migración es más fecuente. Respecto a esto, los autores pasan por alto el efecto de la sobremortalidad masculina. Para 1980, Mina (1986) estima la esperanza de vida al nacer de las mujeres en el Distrito Federal en 71.27, que resulta 6.47 años mayor que la de los hombres. Para la delegación Cuauhtémoc los datos respectivos para las mujeres son: 68.61 y 8.01 años. 
pero entre 1970 y 1980 se aprecia una disminución en sus niveles de crecimiento (Garza y Schteingart, 1984). Asimismo se ha modificado el grado de participación de la población en las actividades productivas y se han producido transformaciones importantes en la estructura sectorial y ocupacional de la mano de obra (Oliveira y García, 1986). Se estima que $42 \%$ de la mano de obra se encuentra en el sector secundario y $52 \%$ en el terciario.

En el momento actual uno de los problemas principales que enfrenta la economía metropolitana es el del empleo, ya que el mercado urbano de mano de obra ha perdido su dinamismo y se ha reducido la capacidad de absorción de fuerza de trabajo en las actividades de corte moderno.

Desde la óptica del empleo, uno de los rasgos que caracterizan al desarrollo capitalista periférico es la diferenciación del mercado de trabajo urbano, que se manifiesta en la existencia y coexistencia de mercados formales e informales de mano de obra (Souza y Tokman, 1976).

Durante la fase de urbanización e industrialización aceleradas, el tránsito de contingentes de población de regiones menos desarrollados hacia la ciudad central, Distrito Federal y AMCM alteró el equilibrio del mercado de trabajo urbano. La oferta de fuerza de trabajo, al interactuar con una demanda insuficiente, que es el reflejo de la incapacidad de absorción de mano de obra de las empresas capitalistas, origina el surgimiento de mercados de trabajo diferenciados.

Al analizar el comportamiento del mercado de trabajo de la ciudad de México y al referirse al comportamiento de los trabajadores por cuenta propia, Muñoz y Oliveira (1976) destacan la presencia de distintos mercados. Alba (1984), reconoce la existencia de dichos mercados. En esta perspectiva se puede afirmar que la fase de urbanización coincide con el fortalecimiento del sector formal y la de suburbanización avanzada con el estancamiento del mismo, y como consecuencia, con el ensanchamiento del sector informal, aunque de manera tentativa dados los problemas de información. Respecto al momento actual, Oliveira y García (1986) apuntan una conclusión similar a la antes expresada.

En los estudios señalados para identificar a los integrantes del sector informal, se han mencionado la relación laboral, el grado de organización de las unidades productivas, la tecnología utilizada, las redes familiares y los niveles de ingreso. Para el caso de México, la Secretaría de Trabajo y Previsión Social, en coordinación con la Secretaría de Programación y Presupuesto, elaboró una encuesta complementaria a la encuesta continua de mano de obra, para definir y delimitar el tipo de población ocupada en las zonas urbanas del país bajo condiciones de informalidad. De 
acuerdo con los resultados, el estereotipo del trabajador informal está dado por las siguientes propiedades: trabajadores jóvenes o de edad avanzada, en su mayoría del sexo femenino, que trabajan por cuenta propia, con bajo capital educativo y niveles de ingreso por debajo del salario mínimo (STPS, 1985). ${ }^{6}$

Aunque en la encuesta de damnificados no se definieron las ocupaciones informales, la captación de los datos según el gobierno, las empresas paraestatales, las empresas privadas, el negocio propio y el ambulantaje permite agruparlos y presentarlos bajo los rubros de los sectores formal e informal. En el primero se incluye al gobierno e industria paraestatal y empresas privadas, en el segundo al trabajador por cuenta propia y a los ambulantes.

Establecer a priori la clasificación de la población ocupada por sectores formal e informal conlleva algunos riesgos; el principal es que por definición se ha denotado como mano de obra informal sólo a los que trabajan por cuenta propia y al ambulantaje. Si bien el autoempleo es uno de los elementos estratégicos para identificar al trabajador informal (Souza y Tokman, 1976), se reconoce que al utilizar sólo este criterio se excluye la posibilidad de reconocer la existencia de la informalidad en el gobierno y en la industria. Se estima que este tipo de trabajo es de $1.3 \%$ en el primero y de $20.5 \%$ en la segunda (STPS, 1985). Lo anterior es la limitante principal del trabajo.

En el cuadro 3 se presenta información derivada del Censo de Población de 1980 y de la encuesta de damnificados. Aunque la información no es del todo comparable, se incluye para tener un punto de referencia sobre la confiabilidad y representatividad de la información. Las mayores discrepancias entre ambas fuentes se presentan en la distribución de la PEA por posición ocupacional y en las tasas globales de participación. Respecto a la primera, las diferencias se aprecian en el grupo de patrones y en el rubro de otros. En este último caso se explica la disimilitud porque el dato censal incluye a los trabajadores familiares no remunerados, los que quedaron fuera de la encuesta, ya que ésta incluye sólo a los trabajadores que declararon recibir sueldo o salario a cambio del trabajo. ${ }^{7}$ En

${ }^{6}$ De acuerdo con el marco conceptual utilizado en la encuesta, la participación de la población en la actividad económica queda dada por la percepción o no de ingresos por concepto de trabajo. A partir del concepto de ingresos se establece la posición en el trabajo y el sector de actividad. Los que no declararon ingresos pasan a formar parte de la población inactiva. En este grupo se incluye a rentistas, pensionados, estudiantes, amas de casa, jubilados e incapacitados temporal o permanentemente.

${ }^{7}$ Hay que subrayar que las preguntas utilizadas en la encuesta para captar la actividad económica entre damnificados difiere de la empleada en el censo de población de 1980, ya que se usaron conceptos y periodos de referencia distintos. El censo empleó la semana anterior a la fecha del levantamiento censal y la encuesta la primera quincena de septiembre, casi un mes antes de realizarse la encuesta. 
cuanto a los niveles de participación, los valores para los hombres y mujeres pueden verse afectados por lo antes dicho, aunque es poco probable que se hayan dado sesgos en la respuesta con sentido opuesto (sobrestimar a los primeros y subestimar a las segundas). Más bien sus valores pueden reflejar las tendencias hacia la disminución que registra la participación masculina y hacia el alza en el caso de la femenina (Morelos, 1972; García, 1984).

CUADRO 3

Comparativo de algunos indicadores sobre la actividad económica

\begin{tabular}{lrrr}
\hline & Censo $^{\mathrm{a}}$ & & Encuesto $^{\mathrm{b}}$ \\
\cline { 2 - 3 } & 1980 & & 1985 \\
\hline Tasa global de actividad (por mil) & 735 & 662 \\
Hombres & 404 & 450 \\
Mujeres & 1000 & 1000 \\
Distribución de la PEA por edad (por mil) & 593 & 584 \\
$12-34$ & 407 & 416 \\
35 y más & & \\
Distribución de la PEA por posición & 1000 & 1000 \\
en la ocupación (por mil) & 49 & 20 \\
Patrón & 630 & 677 \\
Empleado, obrero, peón & 98 & 102 \\
Trabaja por cuenta propia & 223 & $197^{\mathrm{b}}$ \\
Otros & & \\
\hline
\end{tabular}

a Incluye las delegaciones Cuauhtémoc y Venustiano Carranza.

b Ambulantes.

De los 6340 ocupados que arroja la encuesta, $63 \%$ corresponde a los hombres y $37 \%$ a las mujeres, porcentajes muy similares a los que se obtienen en las encuestas continuas de empleo urbano.

Si se atiende a la información recabada sobre el ingreso y escolaridad de los damnificados, el perfil que se obtiene es propio de los integrantes de barrios populares (OIT, 1974). El cuadro 4 pone en claro las diferenciales de ingreso y que la discriminación se da en contra de las mujeres; $28.7 \%$ de las mujeres recibían menos de la mitad del salario mínimo, mientras que en el caso de los hombres era de $\mathbf{1 5 . 7 \%}$. Hasta un ingreso igual a 41280 pesos, los porcentajes para hombres y mujeres fueron de 65.8 y 75.5 por ciento, respectivamente. El mismo panorama se obtiene al comparar los porcentajes para cada uno de los montos de ingreso equivalente en salarios mínimos (véase cuadro 4).

En cuanto a la escolaridad, los datos apuntan en la misma dirección. La proporción de analfabetos es de $3.1 \%$ contra $8.8 \%$ de analfabetas. Con 


\section{CUADRO 4}

Distribución de la población ocupada según monto de ingresos ${ }^{1}$ y sexo ${ }^{2}$ (porcentajes)

\begin{tabular}{crrrrr}
\hline & Hombres & Mujeres & Total & Hombres & Mujeres \\
\hline Ingreso equivalente en & & & & & \\
salarios mínimos & 100.0 & 100.0 & 100.0 & 63.1 & 36.9 \\
Menos de 0.5 S M & 15.7 & 28.7 & 100.0 & 46.9 & 53.1 \\
$0.5-0.8$ S M & 27.1 & 27.1 & 100.0 & 61.8 & 38.2 \\
$0.9-1.1$ S M & 23.0 & 19.7 & 100.0 & 65.4 & 34.6 \\
$1.2-1.4$ S M & 15.3 & 11.3 & 100.0 & 68.7 & 31.3 \\
$1.5-1.9$ S M & 10.8 & 6.2 & 100.0 & 73.8 & 26.2 \\
$2.0-2.9$ S M & 5.2 & 5.3 & 100.0 & 61.3 & 38.7 \\
3.0 y más & 2.9 & 2.4 & 100.0 & 65.7 & 34.3 \\
\hline
\end{tabular}

${ }^{1}$ El salario mínimo mensual de la zona era de 37500 pesos.

2 Elaboraciones con base en los resultados de la encuesta de damnificados.

estudios de primaria - completa e incompleta-, los valores favorecen a la población femenina (50.6\%) frente a $53.0 \%$ de los hombres. En cambio la situación se revierte en el caso de estudios secundarios (véase cuadro 5).

De los datos expuestos se deduce que la estructura de ingresos y el contenido educativo de la población damnificada se caracteriza por el alto grado de concentración de los trabajadores en los estratos más bajos, siendo la más afectada la mujer; lo mismo sucede en el caso de la escolaridad.

Por referirse los datos al total de la población ocupada, no se puede corroborar si los trabajadores informales son los menos educados y los que perciben los más bajos ingresos. Empero, el pérfil señalado coincide con lo que se ha encontrado en el estudio de la informalidad en áreas urbanas (STPS, 1985).

\section{CUADRO 5}

Distribución de la población ocupada según escolaridad y sexo ${ }^{1}$ (porcentajes)

\begin{tabular}{lrrrccc}
\hline & Total & Hombres & Mujeres & Total & Hombres & Mujeres \\
\hline Total & 100.0 & 100.0 & 100.0 & 100.0 & 63.1 & 36.9 \\
Analfabeta & 4.9 & 3.1 & 8.8 & 100.0 & 42.6 & 57.4 \\
Primaria incompleta & 22.5 & 21.8 & 24.0 & 100.0 & 66.0 & 34.0 \\
Primaria completa & 29.7 & 31.2 & 26.6 & 100.0 & 71.5 & 28.5 \\
Secundaria incompleta & 12.5 & 13.7 & 9.9 & 100.0 & 74.6 & 25.4 \\
Secundaria completa & 15.0 & 14.1 & 16.7 & 100.0 & 64.3 & 36.7 \\
Preparatoria o equivalente & 15.4 & 16.1 & 14.0 & 100.0 & 71.1 & 28.9 \\
\hline
\end{tabular}

${ }^{1}$ Elaboraciones con base en los resultados de la encuesta de damnificados. 
Según el cuadro 6, de los 3616 hombres, $65.3 \%$ trabajaban en el sector formal; un porcentaje ligeramente inferior (63.9\%) corresponde a las 2724 mujeres. En el estudio antes citado, el porcentaje de la población masculina que trabaja en ese sector es igual al aquí anotado. El de las mujeres difiere, ya que el estudio consigna $55 \%$. No obstante ser el diferencial mayor, éste está en la dirección esperada.

\section{CUADRO 6}

Distribución de la población según informalidad y por sexo ${ }^{1}$ (porcentajes)

\begin{tabular}{lcccrrr}
\hline & Total & Hombres & Mujeres & Total & Hombres & Mujeres \\
\hline Total & 100.0 & 65.7 & 34.3 & 100.0 & 100.0 & 100.0 \\
Formal & 100.0 & 66.2 & 33.8 & 64.9 & 65.3 & 63.9 \\
Informal & 100.0 & 64.8 & 35.2 & 35.1 & 34.7 & 36.1 \\
\hline
\end{tabular}

${ }^{1}$ Elaboraciones con base en los resultados de la encuesta de damnificados.

\section{CUADRO 7}

Distribución de la población según informalidad por edad y sexo ${ }^{1}$ (porcentajes)

\begin{tabular}{|c|c|c|c|c|c|c|}
\hline \multirow[b]{2}{*}{ Edad } & \multicolumn{3}{|c|}{ Formal } & \multicolumn{3}{|c|}{ Informal } \\
\hline & Total & Hombres & Mujeres & Total & Hombres & Mujeres \\
\hline $12-34$ & 100.0 & 79.9 & 20.1 & $\overline{100.0}$ & 83.3 & $\overline{16.7}$ \\
\hline $35-64$ & 100.0 & 65.7 & 34.3 & 100.0 & 56.6 & 43.4 \\
\hline \multirow[t]{2}{*}{65 y más } & 100.0 & 48.4 & 51.6 & 100.0 & 21.4 & 78.6 \\
\hline & 100.0 & 100.0 & 100.0 & 100.0 & 100.0 & 100.0 \\
\hline $12-34$ & 67.1 & 64.7 & 45.4 & 39.9 & 65.7 & 31.5 \\
\hline $35-64$ & 31.2 & 33.2 & 48.3 & 53.6 & 33.5 & 61.6 \\
\hline 65 y más & 1.7 & 2.1 & 6.3 & 6.5 & 0.8 & 6.9 \\
\hline
\end{tabular}

${ }^{1}$ Elaboraciones con base en los resultados de la encuesta de damnificados.

Otra propiedad del sector informal es que una alta proporción esta constitutida por población joven y trabajadora de edad avanzada. En el cuadro 7 se observa que los hombres se encuentran concentrados $(65.7 \%)$ en el grupo de edad 12-34 y hay un mínimo en el de 65 y más $(0.8 \%)$. En el sector formal los valores para los primeros grupos de edad son muy similares. En el caso de las mujeres en el sector informal, se ubican mayoritariamente en el grupo de edad 35-64 (61.6\%). En el grupo de 65 y más el porcentaje de las mujeres contrasta con el de los hombres (véase cuadro 7). La distribución por edades de la población femenina en el sec- 


\section{CUADRO 8}

Distribución de la población por rama de actividad: sector formal e informal y sexo ${ }^{1}$ (porcentajes)

\begin{tabular}{|c|c|c|c|c|c|c|}
\hline & \multicolumn{6}{|c|}{ Formal } \\
\hline & Total & Gobierno & $\begin{array}{c}\text { Empresa } \\
\text { privada }\end{array}$ & Total & Gobierno & $\begin{array}{l}\text { Empreso } \\
\text { privada }\end{array}$ \\
\hline Total & 100.0 & 26.6 & 73.4 & 100.0 & 100.0 & 100.0 \\
\hline Hombres & 100.0 & 28.6 & 71.4 & 66.2 & 64.5 & 64.2 \\
\hline \multirow[t]{3}{*}{ Mujeres } & 100.0 & 26.9 & 73.1 & 33.8 & 35.5 & 35.8 \\
\hline & \multicolumn{6}{|c|}{ Informal } \\
\hline & Total & $\begin{array}{c}\text { Negocio } \\
\text { propio }\end{array}$ & $\begin{array}{l}\text { Ambu- } \\
\text { lantaje }\end{array}$ & Total & $\begin{array}{c}\text { Negocio } \\
\text { propio }\end{array}$ & $\begin{array}{l}\text { Ambu- } \\
\text { lantaje }\end{array}$ \\
\hline Total & 100.0 & 37.8 & 62.2 & 100.0 & 100.0 & 100.0 \\
\hline Hombres & 100.0 & 38.9 & 61.1 & 64.8 & 66.7 & 71.4 \\
\hline Mujeres & 100.0 & 35.6 & 64.4 & 35.2 & 33.3 & 28.6 \\
\hline
\end{tabular}

${ }^{1}$ Elaboraciones con base en los resultados de la encuesta de damnificados.

tor formal acusa un comportamiento similar al observado en el informal, aunque las cifras son inferiores. Sólo la proporción de mujeres en la edad de 65 y más apunta débilmente a la caracterización que por edad se ha hecho de la informalidad femenina.

En cuanto a la distribución por ramas de actividad, se tiene que la mayoría de los damnificados tenían su puesto de trabajo en la empresa privada, correspondiendo el mayor porcentaje a las mujeres (73.1\%), frente a $71.4 \%$ de los hombres. En el sector informal, $64.4 \%$ de las trabajadoras se dedicaban al ambulantaje. En el caso de los hombres, $61.1 \%$ (véase cuadro 8).

Lo expuesto hasta aquí pone de manifiesto que son las mujeres las peor pagadas y por consiguiente las más explotadas, lo que las convierte en candidatas para engrosar las filas del subempleo invisible, el cual es más frecuente en el sector informal que en el formal. Es posible que el subempleo debido a una remuneración baja sea relativamente mayor en la empresa privada que en el gobierno, ya que en este último son menos frecuentes las violaciones a los tabuladores. En cambio, las empresas privadas pagan en ocasiones salarios que están por debajo del mínimo establecido. Por otra parte, es más probable que en el sector gubernamental el subempleo se explique por la utilización parcial de la calificación o capacidad del individuo.

Lo antes mencionado no constituye en conjunto más que algunas de las manifestaciones del problema de la subutilización de la mano de obra, 
mismo que adquiere mayores visos de gravedad en los sectores informales de las economías en desarrollo.

Con base en los resultados obtenidos en estudios de campo (Souza y Tokman, 1976), se concluye que la migración rural y la proveniente de las ciudades pequeñas y medianas utiliza como vía de acceso al mercado de trabajo urbano al sector informal. Pero debido a la facilidad de entrada que caracteriza a este sector, su importancia relativa tiende a elevarse en periodos de agravamiento de la situación ocupacional.

En las delegaciones Cuauhtémoc y Venustiano Carranza, donde se desarrollan múltiples actividades propias del sector informal, sus integrantes son una mezcla de migrantes viejos y de nativos jóvenes. Dado el gradual despoblamiento que están experimentando ambas jurisdicciones político-administrativas, es de suponer que son los factores de demanda más que los de oferta los que determinan los problemas de empleo.

$\mathrm{Al}$ ocasionar el sismo la pérdida de los lugares de trabajo o del capital de trabajo, o al haber producido lesiones a los trabajadores, se ha agravado la subutilización de la mano de obra en dichas delegaciones.

En el cuadro 9 se presentan las tasas de desempleo que se atribuyen al sismo. ${ }^{8}$ Se debe advertir que hay que interpretar estos resultados con cautela. Se desconoce o, mejor dicho, no hay forma de determinar si la pérdida del empleo fue permanente o sólo temporal. Hubo muchas empresas que por la suspensión de los suministros de agua, de energía eléctrica o de energéticos se vieron en la necesidad de suspender las actividades. El sismo también afectó la actividad industrial y comercial, sobre todo de aquellos locales que se encontraban en áreas a las que se restringía o prohibía el acceso al público.

Tomando en cuenta estas salvedades, los datos de desempleo sugieren que tanto en el sector formal como en el informal, la población más afectada fue la senecta (mayores de 65 años); las tasas de desempleo de las mujeres son sistemáticamente superiores a la de los hombres, y los niveles de desempleo son más elevados en el sector informal que en el sector formal.

Si se consideran las características antes señaladas, es posible que los viejos que se encontraban en la empresa privada y que perdieron su empleo,

${ }^{8}$ De acuerdo con los reportes de los encuestadores, hubo cierta confusión en las respuestas, ya que se identificaba la pérdida de trabajo con la del puesto de trabajo. Pueden plantearse las siguientes situaciones: que la persona hubiera perdido su lugar de trabajo pero no su puesto, que hubiera perdido su puesto por haberse destruido el lugar de trabajo o que hubiera perdido su puesto sin haberse destruido el lugar de trabajo. Por efectos del sismo se estaría frente a los dos primeros casos, pero no en el tercero. Sin embargo, puede haber ocurrido que algunos trabajadores hayan perdido su empleo con fecha anterior al sismo debido a despido, liquidación o jubilación por haberse cerrado la fábrica, trasladado al interior del país o por recorte de personal, tanto en la empresa privada como en el sector gubernamental. 


\section{CUADRO 9}

Tasa de desocupación a causa del $\operatorname{sismo}^{1}$ (por mil)

\begin{tabular}{|c|c|c|c|c|c|c|}
\hline \multirow[b]{2}{*}{ Edad } & \multicolumn{2}{|c|}{ Formal } & \multicolumn{2}{|c|}{ Informal } & \multicolumn{2}{|c|}{ Total } \\
\hline & Hombres & Mujeres & Hombres & Mujeres & Hombres & Mujeres \\
\hline Total & 210 & 316 & 376 & 481 & 258 & 365 \\
\hline $12-34$ & 222 & 302 & 363 & 441 & 255 & 380 \\
\hline $35-64$ & 174 & 323 & 383 & 496 & 251 & 390 \\
\hline 65 y más & 428 & 688 & 438 & 615 & 433 & 655 \\
\hline
\end{tabular}

sobre si había conservado o perdido su empleo.

${ }^{1}$ Elaboraciones basadas en los resultados de la encuesta de damnificados.

\section{CUADRO 10}

Población total y población ocupada y tasas de participación de los damnificados por edad y sexo

\begin{tabular}{|c|c|c|c|c|c|c|}
\hline \multirow[b]{2}{*}{ Edad } & \multicolumn{2}{|c|}{ Total } & \multicolumn{2}{|c|}{ Ocupada } & \multicolumn{2}{|c|}{$\begin{array}{c}\text { Tasa de actividad } \\
\% \\
\end{array}$} \\
\hline & Hombres & Mujeres & Hombres & Mujeres & Hombres & Mujeres \\
\hline Total & 5466 & 6060 & 3616 & 2724 & 66.2 & 45.0 \\
\hline $12-34$ & 3844 & 4030 & 2299 & 1710 & 59.8 & 42.4 \\
\hline $35-64$ & 1394 & 1745 & 1165 & 912 & 83.6 & 52.3 \\
\hline 65 y más & 228 & 285 & 152 & 102 & 66.7 & 35.8 \\
\hline
\end{tabular}

se hayan refugiado en el sector informal. Gran parte de los desocupados en el sector informal, principalmente las mujeres, mantuvieron esa condición sólo temporalmente, dado su estado civil y que muchas de las mujeres damnificadas eran jefes de familia (Rabell y Mier y Terán, 1986).

En el caso de los hombres se puede suponer también que algunos pueden considerase como desocupados temporales y otros más como desocupados ocultos. La presencia de este último tipo de desempleo puede, en parte, ayudar a explicar los niveles relativamente bajos que presentan las tasas de participación por grandes grupos de edad ${ }^{9}$ (véase cuadro 10). Desde esta perspectiva, los desocupados ocultos se encuentran o aparecen junto con la población inactiva debido a que juzgan como inútil concurrir al mercado de trabajo dada la imposibilidad de conseguir empleo.

\footnotetext{
${ }^{9}$ Las tasas de participación obtenidas difieren ligeramente de las conseguidas por Rabell y Mier y Terán debido a que los no especificados se distribuyeron de acuerdo a la importancia relativa de la población en cada grupo de edad. Los valores aquí presentados son más parecidos a la estimación mínima de las autoras mencionadas.
} 
Como explicación alternativa de los bajos niveles de participación masculina, se plantea la tendencia hacia la disminución que desde los años cincuenta se viene dando en las regiones de mayor desarrollo relativo; esta tendencia se acelera en los ochenta debido al desempleo oculto.

Por el contrario, las tasas de participación femenina registran aumentos significativos a partir de 1970. En el caso de la población trabajadora que se encontraba en los albergues y campamentos, el componente tendencial puede ser importante, pero más relevante es el papel que juega en los grupos domésticos la mujer que se incorpora a la actividad cuando el jefe del hogar pierde su empleo.

Ante todo hay que subrayar que el sismo del 19 de septiembre puso en evidencia el poco conocimiento que se tenía sobre la ocupación de sectores populares de la ciudad central. En este sentido la catastrofe resultó importante en cuanto la atención de la población y del gobierno se centró en las delegaciones Cuauhtémoc y Venustiano Carranza. Por otra parte, la información derivada de la encuesta de damnificados sirvió para conocer las condiciones en que vivían y laboraban los afectados por el sismo.

En términos de la ocupación resalta la situación de desventaja en que se encuentran las mujeres adultas y viejas. Dados los niveles de ingreso, se puede decir que el problema del empleo que enfrentan los grupos populares es el del subempleo visible. Existen indicios que hacen suponer que también está presente el desempleo oculto, particularmente en el caso de los hombres.

Aceptando las tasas de desempleo, se puede afirmar que el impacto del sismo en el empleo contribuyó a acentuar la gravedad del problema ocupacional de la ciudad de México. En un estudio reciente sobre el empleo y el crecimiento económico, Urquidi (1986) describe la situación de estancamiento por la que atraviesa la economía mexicana a partir de 1982, así como sus repercusiones en la situación ocupacional global: aumento del desempleo abierto y del subempleo visible. A la misma conclusión llegan García y Tokman (1984). Estos tres autores señalan para el caso de México la creciente importancia del sector informal, la incapacidad de la economía para crear nuevos empleos, así como la caída de los salarios reales.

Si la situación ocupacional que ha sido descrita para la población damnificada se produce en sus rasgos esenciales en otros grupos de población del AMCM, existirían bases para afirmar que la fase de desurbanización estará acompañada por el desempleo y el subempleo en sus diversas modalidades.

Correlativamente a los cambios en la urbanización, la economía metropolitana ha mostrado transformaciones importantes en lo que se refiere 
a la concentración económica y de mano de obra. Ésta se ha visto afectada por la crisis económica, que incide en la pérdida de dinamismo de la planta productiva y en las modificaciones a la estructura del mercado de trabajo urbano. Se estima que el número de establecimientos industriales en 1985 era igual al que existía en 1980, aproximadamente $40 \mathrm{mil}$. Asimismo, el producto interno del área metropolitana ha bajado notablemente. Entre 1982 y 1986 el desempleo ha fluctuado entre 5.0 y 6.5 por ciento, mientras que el subempleo se estima en alrededor de $25 \%$. Paralelo al problema de desempelo y subempleo, se encuentra el de los entrantes a la fuerza de trabajo, cuya presión gravita negativamente en los niveles de empleo. En 1986 la población ocupada en el Distrito Federal se estimaba en 3.6 millones, y el número de personas que se incorporan anualmente a la actividad en aproximadamente $160 \mathrm{mil}$.

En esta perspectiva el problema principal que enfrenta y enfrentará el Distrito Federal es y será el del empleo, ya que el mercado urbano ha perdido su dinamismo al reducirse la capacidad de absorción de mano de obra en las actividades de corte moderno. Por el contrario, han ido en aumento aquellas ocupaciones que se identifican como informales y cuyo aumento en la composición del mercado de trabajo urbano hará más marcada la heterogeneidad estructural de la economía capitalina. Hasta ahora, las políticas del Estado en materia de empleo han dejado de lado a los trabajadores informales, los que con sus propios medios y con el agravio de una fuerte explotación han dado solución a su problema de supervivencia, ya que muchos de ellos no se han visto favorecidos con los programas regionales de empleo y de becas (capacitación) para trabajadores sindicalizados que ha puesto en práctica la presente administración.

\section{Bibliografía}

Alba, Francisco (1984), "Logros y limitaciones en la absorción de la fuerza de trabajo en México", en Demografía y Economía, vol. XVIII, núm. 4(60).

García, Brígida (1984), "Dinámica ocupacional rural y urbana en el sureste de México: 1970-1980", en Demografía y Economía, vol. XVIII, núm. 3(59).

García, Norberto y Víctor Tokman E. (1984), "Transformaciones ocupacionales y crisis", en Revista de la CEPAL, núm. 24, diciembre.

Garza, Gustavo y Martha Schteingart (1984), "Ciudad de México: dinámica industrial y estructuración del espacio en una metrópoli semiperiférica”, en Demografía y Economía, vol. XVIII, núm. 4(60).

Graizbord, Boris (1986), "El crecimiento urbano de México: cambios recientes", ponencia presentada en el II Congreso Iberoamericano de Urbanismo, Tlaxcala, México, abril 21-28.

Korcelli, Piotr (1984), "The Turnaround of Urbanization in Developed Countries", en United Nations, Population Distribution Migration and Development,

Nueva York.

Mina, Alejandro (1986), "Tablas abreviadas de mortalidad por grupo de causas para' 
el Distrito Federal, por sexos, 1980", El Colegio de México (avance de investigación), México.

Morelos, José B. (1972), "Niveles de participación y componentes de cambio de la población activa de México, 1950-1970", en Demografía y Economía, vol. VI, núm. 3(18).

Negrete Salas, María Eugenia y Héctor Salazar Sánchez (1986), "'Zonas metropolitanas en México, 1980", en Estudios Demográficos y Urbanos, vol. I, núm. 1. (s/f), "Dinámica de crecimiento de la población de la ciudad de México, 19001980", por aparecer en Atlas de la ciudad de México.

OIT (1974), "La subutilización de la mano de obra urbana en países subdesarrollados"', trabajo presentado en el Seminario sobre Diseño de Información para Políticas de Empleo celebrado en Brasilia, Brasil, 11 y 13 de septiembre.

Oliveira, Orlandina y Brígida García (1986), "Empleo, desempleo y marginalidad", por aparecer en el Atlas de la ciudad de México.

Rabell, Cecilia y Martha Mier y Terán (1986), "Los damnificados por los sismos de 1985 en la ciudad de México"', Instituto de Investigaciones Sociales, ponencia presentada en el coloquio "El sismo y sus efectos", Ciudad Universitaria, México, 17 y 18 de septiembre.

Secretaría de Trabajo y Previsión Social (1985), Características de la ocupación informal en México, STPS, México.

Souza R., Paulo y Víctor Tokman E. (1976), "El sector informal urbano", en Tokman E., Víctor y Paulo Souza R. (comps.), El empleo en América Latina, México, Siglo XXI.

Stern, Claudio y Rodolfo Corona (1985), "Efectos de la migración rural-urbana sobre las composiciones por edad y sexo de la población: el caso de México", en Estudios Sociológicos, vol. III, núm. 9.

Tarrés, María Luisa (1986). "Crisis and Political Apposition Among Mexican Middle Classes'”, documento presentado a la XI World Congress of Sociology, Nueva Delhi, India, 18-23 de agosto.

Urquidi, Víctor L. (1986) "Empleo y crecimiento econónico", ponencia presentada en la mesa redonda "Bienestar social en tiempos de inestabilidad económica", en El Colegio de México, México, 3 y 4 de septiembre. 


\section{.}

